ARTÍCULOS ORIGINALES

Rev Chil Salud Pública 2019,

Vol 23(1): 21-30

\section{ADHERENCIA A LA INGESTA DE LOS PRODUCTOS DEL PRO- GRAMA DE ALIMENTACIÓN COMPLEMENTARIA DEL ADULTO MAYOR (PACAM) DESDE LA SUBJETIVIDAD DE LOS ADULTOS MAYORES DE LA COMUNA DE HUALPÉN, CHILE}

\author{
UNDERSTANDING OLDER ADULTS' ADHERENCE TO THE CONSUMP- \\ TION OF PRODUCTS PROVIDED BY THE SUPPLEMENTARY FOOD \\ PROGRAM FOR OLDER ADULTS (PACAM) BY EXPLORING THE \\ POINT OF VIEW OF USERS FROM THE MUNICIPALITY OF HUALPÉN, \\ CHILE.
}

\section{RESUMEN}

Introducción. El Programa de Alimentación Complementaria del Adulto Mayor (PACAM) es un programa chileno destinado a prevenir y recuperar el daño nutricional en los adultos mayores. La escasa adherencia de los últimos años motiva este estudio. Objetivo. Comprender cuáles son los motivos de la baja adherencia de las personas mayores a la ingesta de los productos del PACAM (bebida láctea y crema en polvo fortificada llamada 'Años Dorados'), con el fin de proponer alternativas de mejora del programa desde los propios intereses y valoraciones de los usuarios adultos mayores. Método. Es un estudio cualitativo de carácter fenomenológico y hermenéutico enfocado en la interpretación del significado de las experiencias individuales subjetivas. El estudio realizado el primer semestre del 2017, conllevó 20 entrevistas semiestructuradas realizadas a los adultos de ambos sexos beneficiarios del PACAM de la comuna de Hualpén, Chile. Resultados y conclusión. Olor, sabor, consistencia, formas de preparación y cantidad de los formatos son algunos de los elementos organolépticos definidos por las personas mayores, mientras los elementos externos incluyen calidad de vida, red social, influencia de otros usuarios, relación con los profesionales en los servicios de salud, cotidianidad y hábitos.

Palabras claves: Adulto mayor, programa alimentario, adherencia, subjetividad, enfoque de derechos.

\begin{abstract}
Introduction. The Supplementary Food Program for Older Adults (known by the acronym PACAM) is a Chilean program aimed at preventing and/or supporting elderly individuals' recovery from malnutrition. Poor adherence to PACAM in recent years motivates this study. Objectives. To understand the reasons for the low adherence of older adults to the consumption of PACAM products (milk drink and a fortified powdered soup known as "Golden Years (ream"), in order to propose alternatives for improving the program based on the interests and preferences of elderly users. Methods. A qualitative study, with a phenomenological and hermeneutical approach, was conducted, focused on interpreting the meaning of the users' subjective experiences. The study, which was carried out during the first semester of 2017, consisted of 20 semi-structured interviews conducted with older adults of both sexes, who were beneficiaries of PACAM in the Chilean municipality of Hualpén. Results and conclusions: Food odor, taste, consistency, preparation, and quantity were some of the organoleptic properties identified by the subjects, while the external factors included quality of life, social networks, the influence of other users, relationships with professionals in the health centers, daily life, and habits.
\end{abstract}

Keywords: Older adults, Food program, Adherence, Subjectivity, Rights approach.

Artículo recibido el 20/03/2018. Aceptado el 31/07/2018 


\section{INTRODUCCIÓN}

Demográficamente, Chile presenta un cambio progresivo y acelerado de su pirámide poblacional, constituyendo junto a Cuba, Uruguay y Argentina el grupo de países latinoamericanos en transición demográfica avanzada. Este dato se traduce en un aumento considerable de la población adulta mayor. Se estima que para el año 2025, habrá un adulto mayor por cada menor de 15 años y, hacia el 2050, esta cifra aumentaría a 1.71.

El Servicio Nacional Del Adulto Mayor (SENAMA) considera que es necesario una política integral que aborde los desafíos del envejecimiento progresivo de la población en un país donde la brecha de inequidad sigue siendo muy grande $\mathrm{e}^{2-3}$. De este modo, el SENAMA considera que la planificación y distribución de los servicios para los ancianos deben estar enfocadas a dar respuesta a sus necesidades específicas, e idealmente incluir prestaciones que promuevan salud e independencia relativa; incluyendo aquellos con limitaciones que requieran cuidado institucionalizado ${ }^{4-5}$.

Como estrategia para la implementación de las políticas de seguridad alimentaria se desarrollaron los programas de apoyo o asistencia alimentaria, que buscan contribuir a la prevención de la malnutrición por déficit asegurando la disponibilidad y acceso a los alimentos. Por su parte, los países de Latinoamérica y el Caribe implementaron políticas públicas por medio de programas de asistencia alimentaria, enfocados a la población más vulnerable $^{6}$. Su finalidad era garantizar la seguridad alimentaria y nutricional de sus pueblos, considerando los cinco pilares que la componen: disponibilidad, acceso, consumo, aprovechamiento biológico, calidad e inocuidad de los alimentos.

En el caso de Chile, el Ministerio de Salud (MINSAL) crea en 1999 el Programa de Alimentación Complementaria del Adulto Mayor (PACAM). El programa se inició con la entrega mensual de $2 \mathrm{~kg}$ de un alimento precocido Ilamado "Crema Años Dorados". Tras una evaluación inicial, el producto mostró adecuada aceptabilidad por parte de la población beneficiaria; sin embargo, aparentemente, al cabo de unos años su ingesta disminuyó debido a la fatiga generada por el uso reiterado de un producto de sabor definido. En consecuencia, el Ministerio de Salud en conjunto con el Departamento de
Nutrición de la Facultad de Medicina de la Universidad de Chile decidieron explorar nuevas alternativas, desarrollando la Bebida Láctea. El producto mostró una adecuada aceptabilidad y tolerancia, por lo que a partir del 2005 se decide su utilización a nivel nacional, entregando un kilo mensual de "Bebida Láctea", en reemplazo de un kilo de la "Crema Años Dorados". De esta forma, actualmente los beneficiarios del programa tienen derecho al retiro mensual de $1 \mathrm{~kg}$ de Bebida Láctea y $1 \mathrm{~kg}$ de Crema Años Dorados ${ }^{7}$.

A pesar de los beneficios que otorga el Programa, hay una gran cantidad de adultos mayores que dejan de retirar de forma mensual los productos $\mathrm{O}$, en su defecto, los retiran pero no los ingieren. Contamos con algunos estudios previos en Chile donde los usuarios resaltaron los beneficios para la salud y la gratuidad como los elementos más destacables del producto Crema Años Dorados, tras la aplicación de encuestas en el año $2014^{8}$. Con respecto a los motivos por el cual las personas mayores no retiran el producto, estos están relacionados con factores como el disgusto con el producto, limitaciones físicas para ir a retirarlo, o la no necesidad del producto $^{9-11}$. No obstante, se hace necesario una profundización para observar otros elementos no adscritos al producto definidos especialmente desde el conocimiento de los propios beneficiarios ${ }^{12-13}$.

Por consiguiente, este estudio aporta factores nuevos de incidencia en la adherencia a los productos desde la experiencia y valoración de los beneficiarios. Con ellos se puede lograr mayor aprehensión de la situación objetiva y subjetiva, que permita elaborar propuestas más atingentes a la realidad sentida de los adultos mayores y con vistas a integrar el Enfoque de Derechos a un nuevo paradigma para la vejez. Este nuevo paradigma debe desarrollar y garantizar principios de participación, desarrollo integral, adaptación a sus características bio-socio-culturales, limitaciones y potencialidades y, sobre todo, garantizar eficazmente sus derechos a través de las políticas públicas y de desarrollo de programas ${ }^{14}$.

\section{MÉTODOS}

El objetivo planteado fue comprender cuáles son los motivos de la baja adherencia de las personas mayores a la ingesta de los productos del PACAM (Bebida Láctea y Crema Años Dorados), con el fin de 
proponer alternativas de mejora del programa desde los propios intereses y valoraciones de los usuarios adultos mayores. Para ello, se quiso profundizar tanto en los elementos organolépticos como externos al producto, con el fin de aportar y complementar a los estudios previos en Chile.

Se diseñó un estudio cualitativo de carácter fenomenológico-hermenéutico, enfocado en las experiencias subjetivas de los participantes, con el fin de comprender las percepciones de las personas a través de la interpretación que los investigadores hacen del significado que los protagonistas otorgan a los sucesos y vivencias.

El universo se componía de personas mayores beneficiarios del PACAM, que durante el año 2016 no retiraron los productos Bebida Láctea y Crema Años Dorados, o en su defecto los retiraban pero no los consumían. Los adultos mayores formaban parte de los Clubes de Adulto Mayor "Las Maravillas" y "18 de Septiembre", de la comuna de Hualpén, VIII región del Biobío, Chile. También se seleccionaron adultos mayores no vinculados a alguna agrupación, pertenecientes a la misma comuna.

El estudio se desarrolló durante el primer semestre del año 2017 y los participantes (N) fueron 20 personas mayores (11 mujeres y 9 hombres) elegido por intencionalidad representativa de acuerdo a las variables de sexo, edad, pertenencia a grupos asociados; así como criterios de inclusión de voluntariedad, firma del consentimiento informado, relación de cese o no ingesta del producto y otras relacionadas características físico-cognitivas del beneficiario del programa.

La técnica fundamental de trabajo fue la entrevista semiestructurada, flexible, dado que nos interesaba los elementos que aportaba el adulto mayor y la fundamentación que ellos hacían sobre sus respuestas. La técnica escogida nos permitía esa apertura al discurso de los participantes, sin perder de vista los objetivos y las categorías de análisis previo del proyecto: factores internos, externos, otros factores que influían en la adherencia y propuestas de los participantes.

La pauta de entrevista fue revisada por dos investigadores expertos y antes de ser aplicada se tomó con ella una prueba piloto a una adulta mayor de similares características de la muestra.

El proyecto fue sometido a revisión y aprobado por el Comité Ético de la Universidad Católica de la
Santísima Concepción y se tomaron todas las medidas de salvaguarda para la información, confidencialidad y respeto a los participantes.

Las entrevistas fueron grabadas y transcritas en su totalidad para ser analizadas por codificación abierta y lograr el máximo número de categorías en vivo y emergentes, de las que surgieron: 1. Sabor; 2. Consistencia; 3. Olor; 4. Técnicas culinarias; 5. Diversidad de sabores; 6 . Tiempo en casa; 7 . Redes de apoyo; 8. Estado de salud; 9. Recursos económicos; 10. Falta de información; 11. Relaciones interpersonales; 12 . Propuestas; 13. Empoderamiento del equipo multidisciplinario; 14 . Información oportuna; 15. Grado de satisfacción. Estas categorías fueron agrupadas por tema y comparadas aplicando un análisis axial y hermenéutico para conectar sentidos y significados.

\section{RESULTADOS}

\section{Factores organolépticos para el no retiro y no in- gesta de los productos del PACAM.}

Al consultar a los adultos mayores adscritos al Programa, ellos siguen prefiriendo la Bebida Láctea en comparación a la Crema Años Dorados.

De manera que el sabor, el olor, la consistencia, el estado de los productos, la variabilidad y la incompatibilidad de los productos con el estado de salud, son los principales factores internos coincidentes con estudios previos y asociados a la interrupción del retiro y la ingesta de los productos del PACAM. Esto se debe al descontento de la población beneficiaria con las características organolépticas del producto, en especial con el sabor, considerándose procesado y/o sintético, disipándose el sabor original y alejándose del producto natural. Los participantes se refirieron a este como repugnante, desabrida, cargante, entre otros.

En relación a la variedad de sabores disponibles, los adultos mayores consideran que esta variedad es deficiente, refiriendo que las cremas de espárragos y de verduras son las más apetecidas a la hora del retiro y consumo. En cambio, manifiestan su descontento respecto a la crema de lentejas, señalando de forma explícita que es el sabor menos apetecido. Los usuarios comparan la crema con otras cremas artificiales que disponen el mercado, señalando que estas últimas son más sabrosas y aunque tengan que hacer un esfuerzo económico para comprarlas, al- 
Tabla 1. Características de los participantes

\begin{tabular}{|c|c|c|c|c|c|c|}
\hline Participantes & Sexo & Edad & Situación laboral & $\begin{array}{l}\text { Pertenencia } \\
\text { a grupo }\end{array}$ & Adherencia al retiro & $\begin{array}{l}\text { Adherencia a la } \\
\text { ingesta }\end{array}$ \\
\hline 1 & Masculino & 71 & Administrador de empresa & No & Sí & No \\
\hline 2 & Masculino & 76 & Guardia en SAPU & No & No & No \\
\hline 3 & Masculino & 75 & Pensionado & No & Sí & Sí \\
\hline 4 & Masculino & 78 & Pensionado & No & Sí & Sí \\
\hline 5 & Masculino & 67 & Vendedor ambulante & No & No & No \\
\hline 6 & Masculino & 77 & Obrero en la construcción & No & No & No \\
\hline 7 & Masculino & 75 & Jardinero & No & Sí & Sí \\
\hline 8 & Masculino & 70 & Carnicero & No & No & No \\
\hline 9 & Masculino & 80 & Nochero & No & No & No \\
\hline 10 & Femenino & 80 & Pensionado & Sí & Sí & No \\
\hline 11 & Femenino & 73 & Pensionado & Sí & Sí & Sí \\
\hline 12 & Femenino & 84 & Pensionado & Sí & Sí & No \\
\hline 13 & Femenino & 78 & Pensionado & Sí & No & No \\
\hline 14 & Femenino & 74 & Pensionado & Sí & Sí & Sí \\
\hline 15 & Femenino & 78 & Pensionado & Sí & Sí & Sí \\
\hline 16 & Femenino & 84 & Pensionado & Sí & Sí & No \\
\hline 17 & Femenino & 77 & Pensionado & Sí & Sí & Sí \\
\hline 18 & Femenino & 80 & Pensionado & Sí & Sí & Sí \\
\hline 19 & Femenino & 81 & Pensionado & Sí & Sí & No \\
\hline
\end{tabular}

gunos adultos las prefieren.

Otro elemento señalado es la cantidad de producto distribuido. Los productos no son consumidos diariamente, sino entre 2 y 3 veces por semana, por lo que los paquetes quedan en stock en sus casas. Por otro lado, los adultos mayores muestran una preferencia por los productos naturales, e incluso cuestionan por qué el producto que proporcionen los Centros de Salud debe ser procesado y no natural.

En las personas mayores se producen cambios fisiológicos asociados al envejecimiento, destacando las afecciones a nivel digestivo, las cuales se pueden acrecentar tras la ingesta de ciertos alimentos. En relación a lo informado por los participantes, se observó que estos presentaron alteraciones en el sistema digestivo, tales como reflujo gastroesofágico, dolor y/o distención abdominal, al momento de incorporar la Bebida Láctea y la Crema Años Dorados a su alimentación cotidiana.
"La crema tiene un sabor que a uno se le repite después, me daba reflujo" (Participante 5).

"Me dan acidez al tiro y después me da un hambre como a la media hora, y después tengo que andar viendo qué es lo que puedo comer" (Participante 6).

Por lo tanto, desde su percepción, existe una relación directa entre la ingesta de los productos y un efecto negativo sobre el estado de salud, motivo por el cual los adultos mayores interrumpen el retiro y la ingesta de los productos del PACAM. Por consiguiente, cobra relevancia un diagnóstico de problemas gástricos y tolerancia de los usuarios a los componentes del preparado, considerando los nutrientes críticos y aversiones alimentarias de la población adulta mayor, para ofrecerles alternativas que no generen efectos colaterales de daño.

Efectivamente, el Ministerio de Salud a través de 
las SEREMIS de Salud se reserva el derecho de realizar la fiscalización de la composición química, físico-química, microbiológica y sensorial del producto terminado, con la finalidad de verificar que el producto mantenga una óptima calidad y aceptabilidad por el grupo objetivo. Resulta pues fundamental ahondar en los gustos y/o preferencias alimentarias de las personas mayores, con la finalidad de satisfacer sus expectativas y/o necesidades.

Factores externos asociados a la interrupción del retiro e ingesta de los productos del PACAM

Poca disposición a preparar los productos y desconocimiento de cómo hacerlo: En cuanto a los factores externos al producto en sí, encontramos que la habilidad para prepararlo es una constante, ya señalada en otros estudios previos. La preparación o no preparación está ligada al estado de ánimo, a los momentos del adulto mayor y a si tiene a alguien que lo prepare. Habiendo un escaso estimulo por consumir el producto, dado la monotonía y poca atracción, los adultos mayores prefieren comer otra cosa que requiera poca preparación.

Por otro lado, las personas mayores no tienen claridad respecto a la forma adecuada de preparación, señalando que muy esporádicamente reciben información oportuna de parte del equipo de salud o en su defecto se les invita a participar de un taller y/o capacitación. Por otro lado no reciben ningún tipo de material informativo al momento de retirar los productos.

"Ni del momento en que tú vas a retirar los alimentos, no dicen nada, no te explican ninguna cosa, no explican nada. Usted va con su carnet y le entregan no más, nada más" (Participante 1).

Los informantes han mostrado su descontento con el compromiso de los centros en relación a la difusión de información, indicando que les gustaría que hubiese mayor interés y/o disposición de los profesionales.

Escaso estimulo de equipo de salud para el consumo: Las personas entrevistadas valoran las acciones del equipo multidisciplinario de salud, puesto que ven a los profesionales como un referente. Ellos señalan que todas las recomendaciones que les hacen son acogidas en su mayoría. Muchas personas señalaron haber recibido información oportuna de parte del equipo de salud, por ejemplo, con respecto a la edad en que les correspondía acceder a los beneficios del PACAM. Por contraparte, los participantes señalan que cuando no tienen este estímulo, no se motivan al retiro del producto.

"Que te indiquen con más interés y con más tiempo. Porque ahí a la pasadita es como, como que las niñas están cumpliendo" (Participante 2).

Estado de salud mental y demencias asociadas a la edad: Se deben considerar también otros aspectos de salud de los usuarios que inciden en las posibilidades de autonomía que tienen.

"Y lo otro, más grave aún señorita, es que a mí se me borra la película, me olvido, igual que mi señora, cuando le digo "vieja, ime mandaste a buscar los alimentos? -"se me olvido papá" "no importa eso, el otro mes lo vamos a buscarlo-" y el otro mes pasa lo mismo" (Participante 6).

Mejora de la situación económica: Las personas mayores resaltan que una situación económica estable, los permitiría un acceso a alimentos de calidad y no a "esos productos que nos dan". Los informantes lo relacionan con los ingresos económicos percibidos, existiendo una relación directa con la ingesta. Esto quiere decir que prefieren acceder a otros alimentos igualmente procesados (sobres de sopa, cremas, etc.) que ofrece el mercado, cuando su situación económica lo permite. Los adultos mayores ven los productos como un beneficio asistencial que es de gran ayuda en el caso de tener un ingreso económico insuficiente.

"Porque si uno está bien económicamente, uno quiere servirse cualquier otra cosa, pongámosle, cosas de primera, no estos productos que nos dan" (Participante 1).

Situación laboral activa de los adultos mayores: Existen adultos mayores que deben seguir trabajando, dada sus bajas pensiones, con la finalidad de percibir mayores ingresos y sustentar sus gastos. Las pensiones insuficientes ponen al adulto mayor como activo laboral y sujeto a los horarios de trabajo.

"Bien activo, por eso que trabajo actualmente en construcción, bastante lejos trabajamos de las 8:00 de la mañana hasta la 1:00 de la mañana. Tengo que pagar luz, agua, gas, tengo que pagar el cable y con \$120.000 pesos de pensión, no alcanza" (Participante 6). 
Ello contribuye por un lado al cansancio, lo que restringe la movilidad, y también a las condiciones de vida alejadas al estereotipo del adulto mayor; es decir, una vida sedentaria y/o pasiva en espacios relativamente estables y seguros no expuesta excesivos esfuerzos físicos e intelectuales y/o factores estresantes propios de la vida laboral sumado a condiciones precarias de trabajo ${ }^{15}$.

Imaginarios erróneos sobre las características nutricionales del producto: Algunos usuarios señalaron que uno de los factores que ha influido para no consumir el producto es que este engorda. Esta respuesta se señaló en relación a la Crema Años Dorados. No obstante, la crema está pensada para un equilibrio calórico y nutricional. Ello denota que los imaginarios pesan por sobre la información obtenida de los Centros de Salud, lo que probablemente pueda estar conectado al factor de desinformación al que también aluden los beneficiarios.

"La crema, porque no debo comer eso, porque engordo mucho, con la grasa que tienen las cremas" (Participante 16).

Escasas redes sociales: Un factor fundamental que ha influido en la baja adherencia al retiro de los productos del PACAM, es no contar con el apoyo de algún familiar cercano, amigo y/o vecino. Un grupo significativo de adultos mayores viven solos o en su defecto viven junto a familiares, pero no siempre cuentan con el apoyo y/o ayuda de estos.

"De vecinos no, amigos no tengo, de la nieta no tanto, porque trabaja todo el día y llega a dormir no más, en la noche. No tengo la preocupación de nadie" (Participante 14).

Influencia de terceros: Las experiencias de terceros ha sido, también, un factor que ha influido en la baja adherencia al retiro de los productos, demostrando que los adultos mayores se dejan llevar y/o influenciar por los comentarios de terceros, en algunas oportunidades incluso sin haber probado los productos.

"Van a retirarlo, para alimentar los perros, no para alimentarse ellos. Me dicen: no, porque es mala, que voy a estar comiendo esa cuestión, mejor se la doy al perro"

Otros factores: Tenemos que señalar que, en ocasiones, los adultos mayores retiran el producto, pero no lo consumen porque optan por regalarlo a otras personas, percibida como más necesitada, o para las mascotas. Es decir, ellos manifiestan que antes de botarlo, el alimento es regalado. Se encuentra interesante esta repuesta que arroja información para considerar también otros valores adquiridos por ambos productos (leche y crema), distintos del nutricional en la vida de los beneficiarios.

\section{Propuestas de los adultos mayores para mejorar la adherencia a los productos.}

Formato más pequeño del producto: Los participantes señalaron que preferirían una mayor cantidad de Bebida Láctea, en reemplazo de la Crema Años Dorados. Proponen la entrega mensual de 2 kilos de la bebida, debido a que la consumen con mayor frecuencia. En algunos casos, la consumen dos veces al día: al desayuno y a la once, por lo que el producto entregado les alcanza en promedio para dos semanas.

Al mismo tiempo, señalaron que les gustaría que se les entregara una menor cantidad de la Crema Años Dorados, considerando excesiva la distribución de un kilo al mes. Proponen incluso la entrega de 500 gr. dado que la frecuencia de consumo del producto es menor en relación a la Bebida Láctea.

Cambiar el producto por alimentos naturales: En cuanto a la Crema Años Dorados, los adultos mayores preferirían que se les entregara un kilo mensual del ingrediente básico en su estado natural; es decir, un kilo de lentejas, arvejas, etc., con la finalidad de elaborar diversas preparaciones en función de sus preferencias alimentarias.

Modificar el sabor: En cuanto a la Bebida Láctea, la población adulta mayor sostiene que le gustaría que esta tuviera otro sabor, haciendo la comparación con otras leches del mercado. Los nuevos sabores sugeridos consideran: frambuesa, frutilla, plátano, chocolate y vainilla. En cuanto al sabor de la Crema Años Dorados, señalaron que sería ideal que el producto tuviera un sabor más tenue y/o agradable, proponiendo el de carne o en su defecto que fuera como otros productos disponibles en el mercado.

Otras alternativas: En cuanto a las propuestas alternativas señaladas por los entrevistados, estos sostuvieron que les gustaría que se les entregara algún postre y/o galletas para acompañar el té. Por otro lado, no hay precisión respecto a las recetas que les interesaría conocer, manifestando que les gustaría 
Tabla 2. Resumen de factores internos y externos para la interrupción de recogida y consumo de productos del PACAM.

\begin{tabular}{ll}
\hline Factores internos & Factores externos \\
\hline Sabor & Tiempo \\
Variedades de sabores disponibles & Estilos de vida \\
Estado del producto & Estado de salud mental \\
Stock disponible del producto & Calidad de la atención y/o servicio \\
Relación entre frecuencia de consumo y duración del producto & Factores administrativos \\
Comparación con productos del PNAC & Grado de satisfacción con los beneficios que otorga el programa \\
Grado de incompatibilidad con estado de salud & Influencia de experiencias de cercanos \\
------ & Disponibilidad de insumos para elaborar preparaciones \\
\hline
\end{tabular}

incorporar el producto dentro de las preparaciones habituales de su alimentación cotidiana.

\section{DISCUSIÓN}

La mayoría de los aspectos articulados como factores internos se condicen con los estudios anteriores, mostrando ser el sabor, el estado de los productos y la incompatibilidad de los productos con el estado de salud, como los principales factores internos asociados a la interrupción del retiro y la ingesta de los productos del PACAM.

Estos resultados están en parte relacionados con la preparación de los alimentos y las características fisiológicas particulares de las personas. El adulto mayor tiene, comúnmente, cambios fisiológicos en el aparato digestivo que suelen estar asociados al proceso de envejecimiento. Estos no son graves, pero son relevantes ante la presencia de afecciones y deben ser tomados en cuenta a la hora de evaluar por qué se producen los rechazos.

Como hemos señalado, los factores internos no son especialmente novedosos valorando los estudios anteriores al respecto, no obstante, encontramos interesantes los factores externos que aporta una explicación más completa de los motivos por los cuales las personas dejan de tomar el producto PACAM.

Por un lado, encontramos que las personas se mostraron insatisfechos con el actuar y/o compromiso de los profesionales. Esta queja ha sido contemplada en otros estudios en Chile; no obstante, nuestras observaciones añaden otros elementos no evidenciados anteriormente y que están conectados a estos problemas. Más allá de la buena información requerida por los usuarios, estos aluden a factores relacionales, especificando que les gustaría que hubiese mayor interés, disposición y atención por parte de los profesionales en relación a actividades relacionadas con el cuidado de su salud: capacitaciones, consejerías, promoción de la salud, etc. Los entrevistados no solo aluden al qué, sino también al cómo, manifestando que estas actividades fueran realizadas con un real compromiso, empatizando con ellos y sus situaciones.

Los participantes muestran, por lo tanto, que el vínculo entre el envejecimiento y el apoyo social está en relación con la calidad de vida de la población adulta mayor. Ellos observan que la ausencia y/o presencia de apoyo social, sea este positivo y/o negativo, influirá de manera directa o indirecta en aspectos personales. Las redes de apoyo disponibles del adulto mayor son especialmente decisivas a la hora de distribuir sus tareas cotidianas y en el fortalecimiento de su estado de ánimo y de salud, en especial el día que les toca retirar los productos.

Estos factores relacionales, sociales, apoyan la multifactorialidad del fenómeno de adherencia. A partir de la década de los 50, en Chile, se desarrolla un modelo de creencias en salud que surge para explicar las conductas de salud. Este modelo se debía enfocar en detectar las variables apropiadas para diseñar programas de educación para la salud ${ }^{16}$. No obstante, los logros para consolidar una mirada integral, todavía es una asignatura pendiente en el desarrollo de programas que impliquen factores sociales que influyen directamente en las conductas de los pacientes y que puedan definir el perfil que 
cada persona tiene como paciente. Esto contribuiría a que los sujetos no sean estandarizados en los programas nutricionales, es decir, que se realice una valoración de qué tipo de producto complementario debe ser prescrito según las características de cada adulto mayor.

Cabe señalar que parte de los adultos mayores entrevistados no siempre cumplen con el perfil de persona pasiva y/o dependiente, puesto que un segmento importante de esta población contribuye de manera significativa en el bienestar de sus familias, por medio de recursos económicos, apoyo en el cuidado de los nietos o responsabilizándose con las tareas domésticas.

Ello arroja una caracterización social que cuesta identificar con el estereotipo de los usuarios de PACAM: personas pasivas con bajo gasto calórico y escasa autonomía, relacionada con los adultos mayores institucionalizados y dependientes ${ }^{17-18}$ y que no pueden llevar una dieta normalizada y natural.

Recientemente el programa ha tenido una reestructuración que modifica el formato a 500 gramos en el caso de la Crema Años Dorados. Según nuestros resultados a raíz de esta pequeña muestra, la adherencia al Programa de Alimentación Complementaria del Adulto Mayor podría no mejorar significativamente. Ello se debe a que como se ha puesto de manifiesto, los factores internos al producto solo son parte de los motivos de la adherencia a este. Otra gran parte de las limitaciones a la adherencia se debe a factores externos, relacionados con la calidad de vida, el tipo de actividad diaria que realizan, factores económicos, el trato en los servicios, la influencia de otros consumidores y la información disponible.

No queremos pasar por alto la importancia una variable como es la situación de precariedad económica y social de los adultos mayores. Desde el año 2013 al 2016 ha aumentado el porcentaje de personas mayores que trabajan remuneradamente, pasando de un $29.7 \%$ a un $32.4 \%$, respectivamente ${ }^{19}$. Se puede afirmar que cada vez son más las personas que consideran que deben trabajar durante la vejez debido al bajo ingreso.

Estas características de pobreza, escasa red de apoyo social y económico, sumado a la obligatoriedad de mantenerse activo laboralmente ${ }^{15}$, está directamente relacionado con las formas de preparar alimentos y tipo de relación que tienen o pue- den tener con la alimentación. Considerar la actual situación de nuestros adultos mayores, permitiría incorporar esos factores de la vida personal y cotidiana, si, efectivamente, se quiere proponer un proyecto integral de mejora de su calidad de vida. En este proyecto es esencial un enfoque de dere$\operatorname{chos}^{14}$, articulado a las necesidades nutricionales y alimentarias de las personas mayores, además de considerarlos como ciudadanos capaces de decidir y aportar a los servicios que se le ofrecen.

\section{CONCLUSIONES}

La adherencia es un proceso influenciado por múltiples factores relacionados entre sí de forma sistémica ${ }^{20}$ : personales (edad, sexo, trayectoria personal, creencias religiosas y actividad vital), otros de salud mental y física articulados a la vejez, características familiares, situaciones étnicas, redes sociales y económicas, contextos intersubjetivos de relación con los servicios de salud ${ }^{21-23}$ donde la percepción subjetiva de todo ello es primordial24-5. Esto deja en evidencia que la adherencia a un programa de salud es compleja de abordar, puesto que requiere de intervenciones eficaces, útiles y factibles de parte de un equipo multidisciplinario. Este equipo requiere de una mirada holística y personalizada, para cada caso particular, al mismo tiempo. Es por ello, que se considera fundamental que las intervenciones para mejorar la adherencia sean discutidas con el paciente, considerando sus necesidades y problemas desde su subjetividad y vivencia.

Consideramos la pertinencia de un diagnóstico nutricional que permitiría determinar si, efectivamente, hay una necesidad de los productos en sustitución de la alimentación natural, en lugar de considerar que los adultos mayores catalogados como pobres son, por definición, sujetos de atención del beneficio. Especialmente teniendo en cuenta la probabilidad de que sigan activos, con los requerimientos nutricionales que ello conlleva. Las personas mayores se consideran beneficiarias del producto PACAM principalmente por dos indicadores: cumplir la edad y no tener recursos para acceder a una alimentación mejor (ser sujeto de atención de programas sociales y solidarios). La pobreza no debería ser un factor para la perfilación del sujeto beneficiario, dando por sentado que ello los posiciona en una vida inactiva. El acceso a una alimentación natural 
y normalizada, debe de ser garantizado y prioritario en una política pública de salud para el adulto mayor. Por eso debemos destinar esfuerzos en crear alternativas de alimentación natural, que apoye una relación física y culturalmente satisfactoria con los alimentos, que no favorezca la sustitución de la riqueza gastronómica y nutritiva que el adulto mayor puede tener con la comida. El producto PACAM debe complementar una dieta normalizada y solo se puede realizar bajo el análisis de las características particulares de cada adulto.

Como propuesta, se considera viable una pauta diagnóstica nutricional, revisable cada año, que nos permita conocer al usuario en su subjetividad y contexto, e identificar todos aquellos factores que están incidiendo en la vida y salud del adulto mayor en relación a sus necesidades nutricionales: i) factores personales internos al producto: gustos, tolerancia, proceso de alimentos e incompatibilidades; ii) factores conductuales: salud mental, actividad cotidiana, nivel de sedentarismo, tipo de alimentación complementaria, información del producto; iii) y factores sociales: vínculos personales y familiares, confianza y relación con los servicios de salud, redes de apoyo y actividad social. Un diagnóstico nutricional integral de estas características, permitiría ir dando pasos a la integración de todos los aspectos de salud necesarios para tomar decisiones, junto las personas mayores, de forma individual. Todo ello, nos facilitaría asegurar las condiciones de autonomía y normalización de la vida cotidiana, desde una perspectiva de protección y una garantía de los derechos, en los que los servicios públicos de salud tienen un papel fundamental.

Agradecimientos: Les agradecemos sinceramente la participación de todas las personas mayores de la comuna de Hualpén, los cuales entregaron su visión poniendo su mejor voluntad y disponibilidad.

\section{REFERENCIAS BIBLIOGRÁFICAS}

1. Ministerio de Desarrollo Social. Encuesta de Caracterización Socioeconómica Nacional. Santiago. 2015. Recuperado de http://observatorio.ministeriodesarroIlosocial.gob.cl/casen-multidimensional/casen/docs/ CASEN_2015_Resultados_adultos_mayores.pdf

2. Huenchuan S, Rodríguez-Piñero L. Envejecimiento y derechos humanos: situación y perspectivas de pro- tección. CEPAL: Santiago de Chile. 2010.

3. PUC. La IV Encuesta Nacional de calidad de vida en la vejez: Una década de análisis sobre adultos mayores. 2017. Recuperado de http://estudiosdevejez. uc.cl/images/documentos/Libro\%20CHILE\%20Y\%20 SUS\%20MAYORES.pdf

4. Sanhueza M, Castro M, Merino J. Adultos mayores funcionales: Un nuevo concepto en salud Cienc. enferm. [Internet]. 2005 Dic [citado 2018 Jul 31]; 11( 2 ): 17-21. Disponible en: https:// scielo.conicyt.cl/scielo.php?script=sci_arttext\&pi$\mathrm{d}=$ S071795532005000200004\&lng=es. http://dx.doi. org/10.4067/S0717-95532005000200004.

5. Vera M. Significado de la calidad de vida del adulto mayor para sí mismo y para su familia. Anales de la Facultad de Medicina [Internet]. [S.I.] 2012. Feb. [citado 2018 jul 31] 68 (3):284-290. Disponible en: http://revistasinvestigacion.unmsm.edu.pe/index.php/ anales/article/view/1218.

6. Almanza O. Programas de apoyo/asistencia alimentaria de Colombia y países de América Latina-México, Bolivia, Chile. Universidad Nacional de Colombia: Bogotá. 2013.

7. Lastra M, Arias M, Ibaceta A et al. Difusión de programa Nacional de Alimentación complementaria para adultos mayores, mediante actividades prácticas dirigidas a adultos mayores hospitalizados en unidad de ortogeriatría. Recuperado de: https://www.sociedadpoliticaspublicas.cl/archivos/BLOQUE_ST/Salud/ Difusion_de_Programa_Nacional_de_Alimentacion_ Complementaria_para_Adultos_Mayores.pdf

8. Ayala, J. (2008). Estudio nacional de aceptabilidad y consumo de los productos de los Programas Alimentarios del Ministerio de Salud: "Mi Sopita", "Purita Mamá" y "Crema Años Dorados". Facultad de Medicina, Universidad de Chile.

9. Almanza O. Programas de apoyo/asistencia alimentaria de Colombia y países de América Latina-México, Bolivia, Chile. Universidad Nacional de Colombia: Bogotá, Colombia. 2013.

10. Silva R, Moya O. Preferencias de consumo de los alimentos del PACAM en dos CESFAM. Región Metropolitana. XXVII Jornadas Chilenas de Salud Pública. 2008. Recuperado de: http://campusesp.uchile. cl:8080/dspace/bitstream/handle/123456789/183/ prom_51.pdf?sequence $=1$ \&isAllowed $=y$

11. Masi C, Atalah E, Análisis de la aceptabilidad, consumo y aporte nutricional del programa alimentario del adulto mayor. Rev. méd. Chile [Internet]. 2008 Abr [citado 2018 Jul 31] ; 136 ( 4 ): 415-422. Disponible 
en: https://scielo.conicyt.cl/scielo.php?script=sci_arttext\&pid=S003498872008000400001\&lng=es.http:// dx.doi.org/10.4067/S0034-98872008000400001 .

12. Blair T, Minkler M. Participatory Action Research with Older Adults: Key Principles in Practice. The Gerontologist 2009 jun [citado 2018 Jul 31]; 49 (5): 651662. Disponible en https://www.ncbi.nlm.nih.gov/ pubmed/19491353

13. Guerrero-Castañeda R, Oliva Menezes TM, Ojeda-Vargas MG. Acercamiento al escenario de estudio fenomenológico en México: relato de experiencia. Revista Gaúcha Enfermagem [Internet]. [citado 2018 Jul 31]; 2017; 70 (4):885-90. Disponible en http:// seer.ufrgs.br/index.php/RevistaGauchadeEnfermagem/article/view/67458

14. Huenchuan, S. Los derechos de las personas mayores. Santiago: CEPAL. 2011.

15. Vives A, Molina A, Nora G, González F, Envejecimiento y trabajo en Chile: propuesta para el monitoreo de la salud laboral. Seminario realizado el 10 de noviembre de 2016, Santiago de Chile.

16. Rosentock I, Strecher V. Becker H. Social Learning and the Health Belief Model. Health Education \& Behavior [Internet]. 1988 feb [citado 2018 Jul 31]; 15 (2): 175 -183 .

17. Lucena $O$, Carneiro $S$, Oliveira $A$ et al. O envelhecimento ativo sob o olhar de idosos funcionalmente independentes. Rev Esc Enferm USP [Internet]. 2010; [citado 2018 jul 31]; 44 (4): 1065-9. Disponible en http://www.scielo.br/pdf/reeusp/v44n4/30.pdf

18. Lastra M, Arias M, Ibaceta A, Difusión de programa Nacional de Alimentación complementaria para adultos mayores, mediante actividades prácticas dirigidas a adultos mayores hospitalizados en unidad de ortogeriatría. Disponible en https://www.sociedadpoliticaspublicas.cl/archivos/BLOQUE_ST/Salud/ Difusion_de_Programa_Nacional_de_Alimentacion_ Complementaria_para_Adultos_Mayores.pdf

19. Ministerio de Desarrollo Social. Encuesta de Caracterización Socioeconómica Nacional. Santiago. 2015. Disponible en http://observatorio.ministeriodesarroIlosocial.gob.cl/casenmultidimensional/casen/docs/ CASEN_2015_Resultados_adultos_mayores.pdf

20. Bronfenbrenner U. Ecological models of human development: In international Encyclopedia of education, Oxford: Elsevier 1994. P.37-43.

21. Rodríguez A, Álvarez L, Quevedo F. Necesidades percibidas de atención de salud al adulto mayor desde una perspectiva de género. Rev Cubana Med Gen Integr [Internet]. 2009 Dic [citado 2018 Jul 31]; 25( 4 ): 65-76. Disponible en: http://scielo.sld.cu/scielo.php?script=sci_arttext\&pid=S0864-21252009000400007\&lng=es.

22. Moreno González A, Incidencia de la actividad física en el adulto mayor. Revista Internacional de Medicina y Ciencias de la Actividad Física y el Deporte [Internet] 2005 [citado 2018 Jul 31]; 5 (20); 222-237. Disponible en http://cdeporte.rediris.es/revista/revista20/ artvejez16.htm

23. Montes V. Redes de apoyo social de personas mayores: elementos teórico-conceptuales. Santiago: CEPAL. 2003.

24. Muñoz C. Bienestar subjetivo y actividad social con sentido histórico en adultos mayores Deporte [Internet] 2013 [citado 2018 Jul 31]; 18 (2):13-26. Disponible en http://www.scielo.org.co/pdf/hpsal/v18n2/ v18n2a02.pdf 